\title{
MGMT methylation in diffuse large B-cell lymphoma: validation of quantitative methylation-specific PCR and comparison with MGMT protein expression.
}

\author{
Uccella S, ${ }^{1}$ Cerutti R, ${ }^{1}$ Placidi C, ${ }^{1}$ Marchet S, ${ }^{1}$ Carnevali I, ${ }^{1}$ Bernasconi B, ${ }^{1}$ Proserpio I, ${ }^{2}$ Pinotti G, ${ }^{2}$ Tibiletti \\ $M G,{ }^{3}$ Furlan $D,{ }^{1}$ Capella $C .{ }^{1}$ \\ 1 Dept of Human Morphology, Unit of Pathology, University of Insubria-Ospedale di Circolo, Varese, Italy; \\ 2 Dept of Oncology, Ospedale di Circolo, Varese, Italy; \\ 3 Dept of Pathology, Ospedale di Circolo, Varese, Italy
}

Correspondence to: Dr S Uccella, Dept of Human Morphology, Unit of Pathology, University of Insubria, Via O.

Rossi, 9, 21100 Varese, Italy; silvia.uccella@uninsubria.it

Accepted 6 April 2009

\section{ABSTRACT}

Purpose: The molecular pathogenesis of gastroenteropancreatic endocrine tumors (ETs) is still largely unknown. The purpose of this work was a molecular characterization of 38 gastroenteropancreatic ETs with respect to the primary site and to the morphofunctional profile, pointing out useful diagnostic or prognostic molecular markers.

Experimental Design: Twenty-four well-differentiated ETs or carcinomas (WDET/Cs; 11 pancreatic, 3 gastric, and 10 intestinal) and 14 poorly differentiated endocrine carcinomas (1 pancreatic, 6 gastric, and 7 colorectal) were microallelotyped using 38 polymorphic microsatellite markers covering chromosomes 1,3 , $5 q, 6,11,17$, and 18 .

Results: Regardless of the primary site, a significantly higher percentage of allelic imbalances (Als) was observed in poorly differentiated endocrine carcinomas than in WDET/Cs $\left(P{ }_{-} 0.012\right)$, except for 3 of 8 nonfunctioning pancreatic endocrine tumors and 1 colorectal WDEC, exhibiting multiple Als on chromosomes 1, 3, 6, and 11. A strong positive correlation between Al percentage and Ki-67

proliferation index was detected considering both the whole series of ETs $\left(P{ }_{-} 0.004\right)$ and the group of WDET/Cs alone $\left(P_{-}\right.$0.011). The survival analysis showed a positive correlation between low percentage of $\mathrm{Al}$ and longer survival $\left(P_{-}{ }_{0} 0.01\right)$. No recurrent Als at specific chromosomal regions were identifiable with respect to the primary site.

Conclusions: The malignant progression of endocrine tumors seems to be associated with complex allelotypes and chromosomal instability. Although no specific molecular markers of malignancy can be defined with certainty, the ploidy status and the degree of chromosomal derangements appear to be the most informative genetic factors with prognostic significance.

Source:

Dept of Human Morphology, Unit of Pathology, University of Insubria-Ospedale di Circolo Via O. Rossi, 9, Varese 21100, Italy. silvia.uccella@uninsubria.it 